\title{
Water Jet Guided Laser Cutting of Silicon Thin Films Using 515nm Disk Laser
}

\author{
Eva-Maria Heilmann ${ }^{*}$, Albrecht Seidl ${ }^{* *}$ and Ralf Hellmann ${ }^{*}$ \\ *University of Applied Sciences Aschaffenburg, Wuerzburger Strasse 45, D-63743 Aschaffenburg \\ E-mail: ralf.hellmann@h-ab.de \\ ** Schott Solar Wafer GmbH, Carl-Zeiss-Strasse 4, D-63755 Alzenau
}

\begin{abstract}
We report on a comprehensive study of water jet guided laser cutting of polycrystalline silicon thin films using a pulsed $515 \mathrm{~nm}$ disk laser. The optimization of the cutting process of polycrystalline silicon wafers having a thickness of $200-300 \mu \mathrm{m}$ is based on a Design of Experiments (DOE) approach taking into account process parameters such as laser power, cutting speed, laser repetition rate, water pressure and nozzle diameter, respectively. The targeted response of the DOE is defined by both the quality as well as the productivity of the cutting process. While the former is measured by the mechanical strength and the chipping of the wafers, the latter is determined by the ablation depth and the cutting kerf cross-sectional area. Our analysis identifies the most influencing parameters on the cutting process, provides insight into the interactions of these parameters and, finally, delivers an optimized process parameter window for water jet guided laser cutting of thin silicon films.
\end{abstract}

DOI:10.2961/jlmn.2011.02.0013

Keywords: water jet guided laser cutting, silicon thin film, Design of Experiment, disk laser

\section{Introduction}

Water jet guided laser cutting using pulsed lasers has recently attracted considerable interest [1-5]. This technology is associated to valuable advantages in laser micromachining as compared to the conventional dry laser based cutting processes with coaxial gas assist. These advantages are, e.g., a significantly reduced heat affected zone due to the cooling of the interaction zone by the continuous water jet in between the laser pulses, an efficient removal of molten material by the water jet, reduced surface contamination by debris, highly parallel kerf walls, and a large working distance determined by the stable laminar water jet, respectively $[3,6]$. The latter, in turn, eliminates the need of any focus control that is typically needed in dry laser cutting to hold the relative position of the focal point and the work piece within the Rayleigh length of the laser source. These advantages have been proven to lead to an excellent cutting quality $[7,8]$.

Using pulsed diode pumped solid state lasers the potential of water jet guided laser cutting has been demonstrated for various materials and applications, such as cutting and grooving of silicon, gallium arsenide and silicon carbide wafers $[6,9,10]$, cutting of thin stainless steel sheets [1], copper foils and polyimide films with cupper plating $[6,10]$, and scribing of low temperature co-fired ceramics [10,11]. From a market point of view, these applications adress industrial fields such as the semiconductor and electronics industry (e.g. the solar cell and high brightness LED market), the medical, tooling and the watch industry, respectively $[1-3,10]$. The cited reports, however, focus on the demonstration of the successful implementation of the technology and do not detail any optimization of the process.

Here, we report on a comprehensive study of water jet guided laser cutting of polycrystalline silicon thin films having a thickness of $200-300 \mu \mathrm{m}$. Similar as the above mentioned applications the cutting process of silicon thin films is governed by the laser while the water jet is responsible for the material removal out of the cutting kerf and of its cooling. Please note that in our experiments the water jet produces only a mechanical strength of $0.17 \mathrm{~N}$. The optimization of the cutting process is based on a DOE approach. The response of the DOE is well defined by the quality and productivity of the cutting process. The former is quantitatively determined by the mechanical strength and the chipping of the silicon wafers and the latter is measured by the ablation depth and the cross-sectional area of the cutting kerf.

Within the DOE approach we take into account the most influencing cutting parameters such as laser power, cutting speed, laser repetition rate, water pressure and nozzle diameter, respectively. As a result, we identify an optimized parameter window with regard to the targeted response (quality and productivity).

\section{Experimental}

\subsection{Polycrystalline wafers}

The polycristalline silicon wafers have been produced by the edge-defined film-fed growth technology (EFG). Details of this technology have been published by the authors and others $[12,13]$. Using EFG, up to seven meter long, thin $(200-300 \mu \mathrm{m})$ octagonal or dodecagonal silicon tubes can be produced. Out of each side of the polygonal tube a $125 \times 125 \mathrm{~mm}^{2}$ square wafer is laser cut and further processed to a solar cell. To maintain a sufficient quality of the cutting edges, usually a multiple pass cutting process is required. 


\subsection{Laser system}

Throughout our study we used a commercial water jet guided laser cutting system (Synova Microjet ${ }^{\circledR}$ ) equipped with a fiber coupled $515 \mathrm{~nm}$ pulsed disk laser delivering an averaged output power of $400 \mathrm{~W}$ at $100 \mathrm{kHz}$ (Trumpf TruMicro 7250). The principle of water jet guided laser cutting has been published in Ref. $[1,14,15]$. As the absorption in water is lowest around 500nm, using a laser in this spectral range is advantageous. In addition, the absorption in silicon is considerably higher by several orders of magnitude in this spectral range as compared to the near infrared (the spectral range of the fundamental emission of solid state lasers) [16].

Own power measurements revealed fiber losses of about $10 \%$ and additional lossed by the optical head of the cutting system and the water jet of about $20 \%$. Hence about $70 \%$ of the laser delivered power is available for laser cutting.

\subsection{Design of Experiment (DOE)}

For the DOE a commercially available software package $\left(\mathrm{JMP}^{\circledR}\right.$ by SAS) was employed. The relevant parameters used in the optimization process are summarized in Table 1, which define the input parameters for the DOE. To get acquainted with the process, the DOE consists initially of a Screening Design, based on which the most influencing parameters on the response are identified. Subsequently, within a Response Surface Design possible interactions between diffeent parameters are examined and the optimized parameter sets are determined. Finally, the calculated optima are further examined with regard of the optimized quality and productivity (response).

\subsection{Responses}

\subsubsection{Quality}

The cutting quality in our study is defined firstly by the mechanical strength of the wafers and secondly by the chipping at the backside of the laser cut wafers. The mechanical strength of the wafers is of crucial importance with respect to subsequent process steps of the wafer within the solar cell manufacturing. As the laser based cutting process is a thermal process, the thermal stress although reduced by the water jet results in mechanical strain leading to microcracks in polycrystalline silicon. The generated micro-cracks in turn lead to a reduction of the mechanical breaking strength, which is tested by a four-bar-bending test [12].

In this test, the thin silicon film is strained by compression. The pressure is caused by four parallel bars. A schematic design is shown in Figure 1.

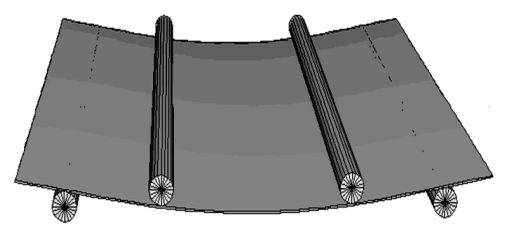

Fig. 1 Four-bar-bending test [12]
Table 1 Input parameter

\begin{tabular}{lll}
\hline Parameter & Range & Unit \\
\hline Wavelength & 515 & $\mathrm{~nm}$ \\
Power (max.) & $35-400$ & $\mathrm{~W}$ \\
Power water jet & $25-275$ & $\mathrm{~W}$ \\
Repetition rate & $5-100$ & $\mathrm{kHz}$ \\
Water pressure & $100-500$ & $\mathrm{bar}$ \\
Nozzle diameter & $60,80,100$ & $\mu \mathrm{m}$ \\
Velocity & $10-500$ & $\mathrm{~mm} / \mathrm{s}$ \\
\hline
\end{tabular}

Chipping refers to material outbreaks at the wafer backside along the cut kerf. To quantitatively assess the chipping, the wafer backside is illuminated and the brightness maxima of the reflection resulting from the chipping are counted using a photo editor. Lower chipping refers to higher quality of the cut kerf.

\subsubsection{Productivity}

From a productivity point of view, the effective cutting speed and the ablation efficiency are of interest. Both parameters can be calculated by measuring the ablation depth and the kerf cross-sectional area.

The theoretical effective cutting speed is given by

$$
v_{\text {eff }}=\frac{A \cdot v}{d}
$$

with the ablation depth $\mathrm{A}$, the velocity $\mathrm{v}$, and the wafer thickness $d$, respectively. The ablation depth describes the average depth $\mathrm{T}$ of the first three passes (p).

$$
A=\frac{T}{p}
$$

The ablation efficiency can be calculated by [17]:

$$
\eta=\frac{\Delta H_{t h, S i}}{\Delta H_{e x, S i}}
$$

The formula describes the ratio of the theoretical energy required to melt $1 \mathrm{~mm}^{3}$ of silicon $\left(\Delta \mathrm{H}_{\mathrm{th}, \mathrm{Si}}\right)$ and the experimental ablation efficiency $\Delta \mathrm{H}_{\mathrm{ex}, \mathrm{Si}}$. The latter is composed of laser power $\mathrm{P}$, velocity $\mathrm{v}$, kerf cross-sectional area $\mathrm{A}_{\mathrm{gr}}$ and the absorption coefficient $\alpha$ of silicon at the laser wavelength. For polycrystalline silicon $\Delta \mathrm{H}_{\mathrm{th}, \mathrm{Si}}$ is $50 \mathrm{~J} / \mathrm{mm}^{3}$ [17] and $\Delta \mathrm{H}_{\mathrm{ex}, \mathrm{Si}}$ can be experimentally determined according:

$$
\Delta H_{e x, S i}=\frac{\alpha P}{A_{g r} v}
$$

Using these values in our study, $\eta$ is calculated and used to evaluate the efficiency of the cutting process.

\section{Results and Discussion}

\subsection{Screening Design (SD)}

To get acquainted with the investigated process, the DOE starts with a SD. As the cutting process is governed by a large number of influencing parameters, the SD also serves to separate those variables with major influence from those 
with minor influence on the response. The latter can then be kept constant during the forthcoming study, i.e. the Response Surface Design (RSD) [18].

To evaluate the influence of the parameters given in Table 2 and to determine the limits of these parameters with respect to a feasible process, we have examined a series of laser cuts in the parameter range given in Table 2 (Screening Design). As a result, the parameter range is reduced to those values that will be further used in the RSD (Table 2).

In our experiments it turns out that the nozzle of the water jet laser system, particularly for smaller nozzle diameters, is sensitive to high laser powers, resulting in nozzle cracks. In addition, as shown in Figure 2 at higher laser powers we observe instabilities of the cutting process and a significant heat affected zone, due to an insufficient cooling rate at power levels beyond $100 \mathrm{~W}$. As a result of our analysis, we therefore limit the maximum laser power for the RSD to $100 \mathrm{~W}$ and the nozzle diameter to $80 \mu \mathrm{m}$, as the later leads to the highest values of the breaking strength.

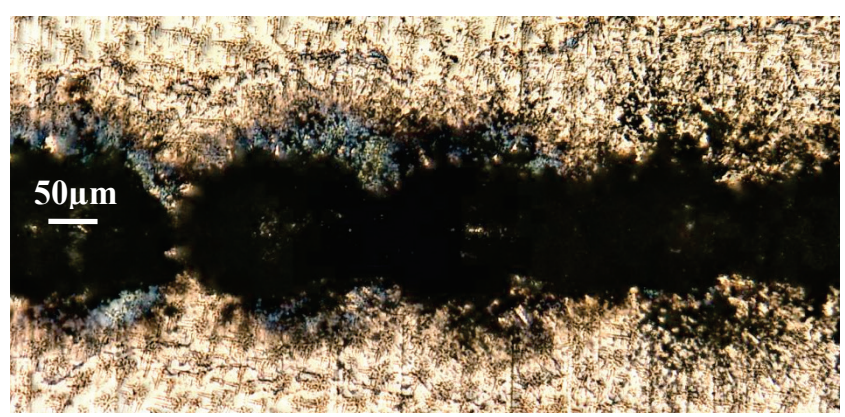

Fig. 2 Instability of the cutting process and heat affected zone at $150 \mathrm{~W}$ laser output power.

Furthermore, the SD reveals that a water pressure of about 330 bar leads to wafer damages, e.g. broken wafers or microcracks. Hence, in the RSD the water pressure is evaluated only in a range between 100-330bar.

As the pulse energy and pulse duration decrease with increasing repetition rate, we find within the SD that efficient material separation is starting at $50 \mathrm{~W}$ at a repetition rate of $55 \mathrm{kHz}$. At maximum repetition rate $(100 \mathrm{kHz})$ a minimum laser output power of $70 \mathrm{~W}$ is required.

To guarantee an efficient material separation, the cutting velocity should not fall below $100 \mathrm{~mm} / \mathrm{s}$. For lower velocities, the process stops almost completely, which might be attributed to a deformation of the water jet impinging onto the steeper cutting edge at lower cutting velocities.

Table 2 summarizes the parameter ranges examined in the $\mathrm{SD}$ and those used in the forthcoming RSD. In total the SD encompassed 21 individual experiments to identify the influence of the examined parameters and to limit the parameter range.

\subsection{Response Surface Design (RSD)}

Based on the results of the $\mathrm{SD}$, the DOE is extended to a RSD by ten further experiments to identify possible quadratic dependencies. The parameter ranges for the RSD are also shown in Table 2, which includes the factors laser output power, repetition rate, water pressure, nozzle diameter and velocity. The parameter levels are illustrated in Table 3.
The objective is to determine the optimized parameter sets for both quality and productivity, respectively.

\subsubsection{Productivity measurements}

In order to achieve high operational throughputs, the effective cutting speed and the ablation efficiency are determined during the RSD by measuring the ablation depth and the kerf cross-sectional area for a series of laser cuts.

A typical cut kerf (single pass) as measured with a digital microscope is illustrated in Figure 4. Here, the kerf width

Table 3 Parameter range used for the Screening Design and subsequent test plans (Response Surface Design)

\begin{tabular}{lll}
\hline $\begin{array}{l}\text { Parameter } \\
\text { (DOE factors) }\end{array}$ & $\begin{array}{l}\text { Screening } \\
\text { Design }\end{array}$ & $\begin{array}{l}\text { Response } \\
\text { Surface Design }\end{array}$ \\
\hline Power water jet & $25-275 \mathrm{~W}$ & $25-100 \mathrm{~W}$ \\
Repetition rate & $5-100 \mathrm{kHz}$ & $5-100 \mathrm{kHz}$ \\
Water pressure & $100-380 \mathrm{bar}$ & $100-330 \mathrm{bar}$ \\
Nozzle diameter & $60,80,100 \mu \mathrm{m}$ & $80 \mu \mathrm{m}$ \\
Velocity & $10-500 \mathrm{~mm} / \mathrm{s}$ & $100-500 \mathrm{~mm} / \mathrm{s}$ \\
& & \\
\hline
\end{tabular}

Table 2 Parameter levels used for the experimental study

\begin{tabular}{ll}
\hline $\begin{array}{l}\text { Parameter } \\
\text { (DOE factors) }\end{array}$ & Levels \\
\hline Power water jet & $25,55,70,90,100,150,200,275$ \\
Repetition rate & $10,55,100$ \\
Water pressure & $100,215,250,330,380$ \\
Velocity & $100,200,250,300,350,400,500$
\end{tabular}

as determined at the top of the kerf is $76.4 \mu \mathrm{m}$. Throughout our study we found that the kerf width decreases with increasing repetition rate. At $100 \mathrm{kHz}$ it is comparable to the used nozzle diameter of $80 \mu \mathrm{m}$ and at $10 \mathrm{kHz}$ the kerf width is about $160 \mu \mathrm{m}$. In addition, lower repetition rates lead to significant heat affected zones due to high pulse energies. Therefore, we limit the repetition rate in the range between $55 \mathrm{kHz}$ and $100 \mathrm{kHz}$.

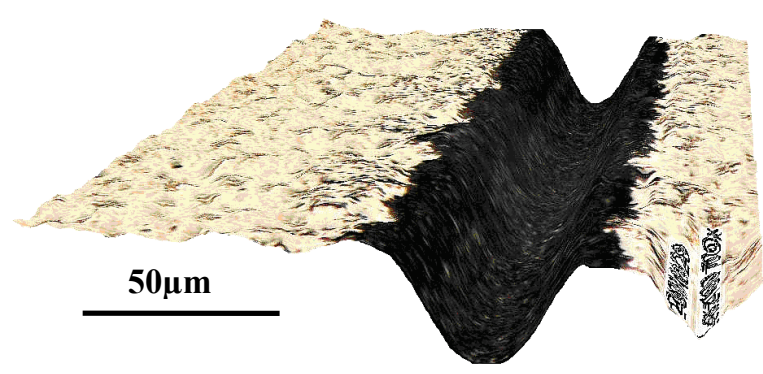

Fig 4 Digital microscope illustration of a cut kerf (single pass) at $215 \mathrm{bar}$ water pressure, $71,7 \mathrm{~W}$ laser output power, $100 \mathrm{kHz}$ repetition rate and $200 \mathrm{~mm} / \mathrm{s}$ velocity. The width of the $\mathrm{v}$-shaped cut kerf is about $76.4 \mu \mathrm{m}$ at its top.

In order to determine the highest effective cutting speed, Figure 5 shows the 3-D diagram of the effective cutting speed versus laser output power and repetition rate. 
Generally, 3-D diagrams serve to visualize the response (e.g. effective cutting speed or ablation efficiency) as a function of two parameters, while the remaining parameters are kept constant. Thus, interactions between two parameters are investigated. For this analysis the cutting velocity was pre-set to $500 \mathrm{~mm} / \mathrm{s}$.

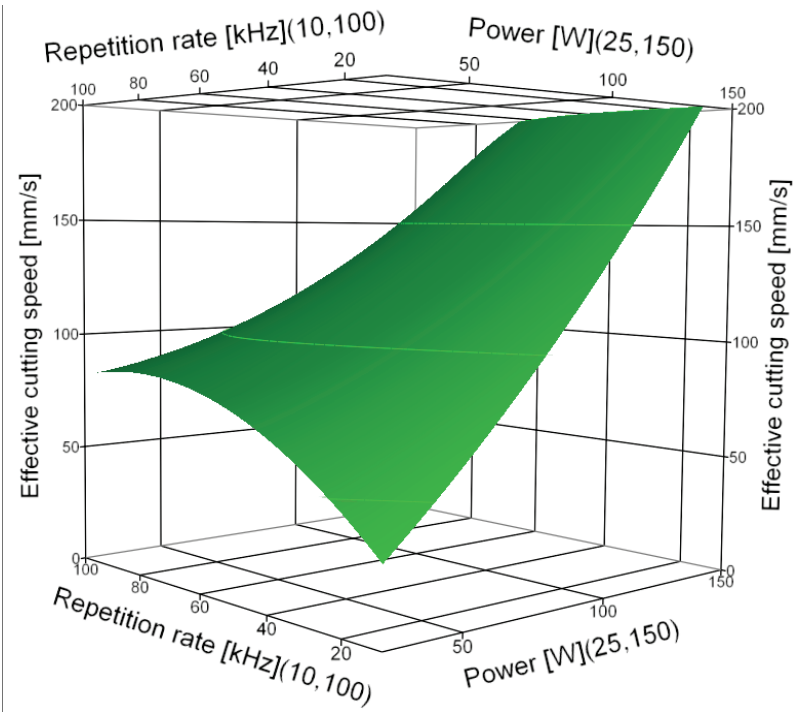

Fig. 5 3-D simulation of the effective cutting velocity as a function of laser output power and repetition rate (pre-set cutting velocity of $500 \mathrm{~mm} / \mathrm{s}$ ).

A contour diagram as shown in Figure 6 is derived from the 3D-diagram, in which each line corresponds to a cut through the surface of the 3D-plot.

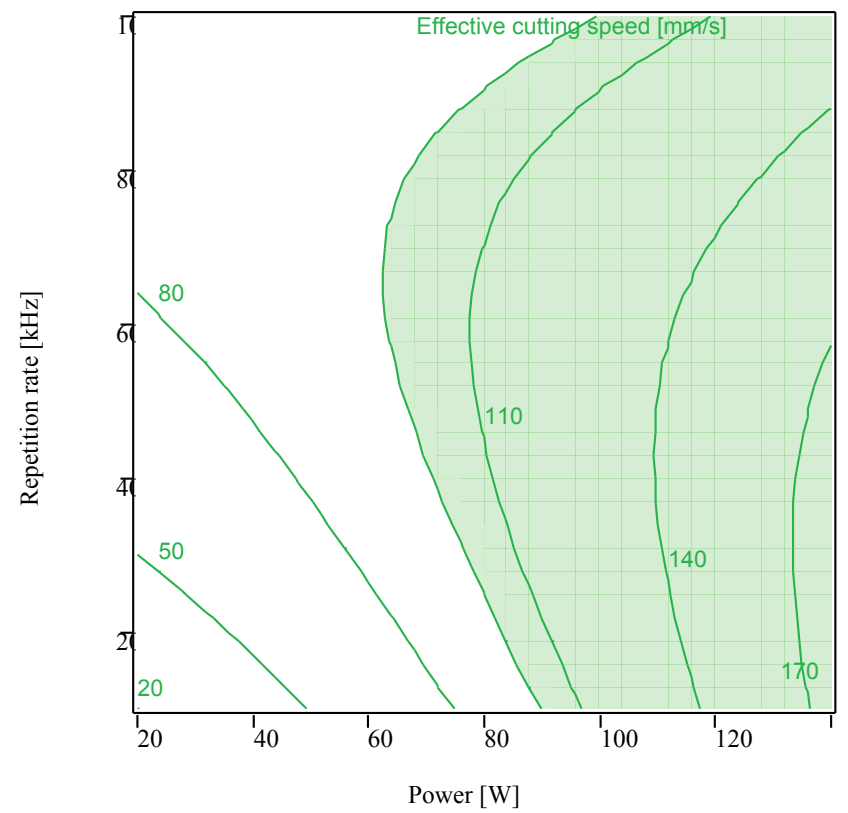

Fig. 6 Preferable effective cutting speed about $100 \mathrm{~mm} / \mathrm{s}$ (marked area) versus laser output power, repetition rate and a pre-set cutting velocity of $500 \mathrm{~mm} / \mathrm{s}$.

The green highlighted area in Figure 6 illustrates the parameter regime of laser output power and repetition rate to obtain a calculated effective cutting speed of at least
$100 \mathrm{~mm} / \mathrm{s}$. Based on large wall thickness variations produced from the EFG procedure and the subsequent increase of passes, in practice an effective cutting speed of $75 \mathrm{~mm} / \mathrm{s}$ is desirable. Hence, a theoretical effective cutting speed of $100 \mathrm{~mm} / \mathrm{s}$ should be achieved. Taking into account the above described power limitations to $100 \mathrm{~W}$, Figure 6 reveals that moderate powers of 50 to $100 \mathrm{~W}$ and mean repetition rates lead to preferable effective cutting speeds.

Based on our measurements of the cross sectional areas of the cutting kerf, we have determined the ablation efficiency $\eta$ by measuring $\Delta \mathrm{H}_{\mathrm{ex}, \mathrm{Si}}$ acccording Formula 3 and 4 . The results are shown in Figure 7 for different parameter combinations in the velocity range between 100 and $500 \mathrm{~mm} / \mathrm{s}$ and repetition rates up to $100 \mathrm{kHz}$. We found maximum ablation efficiencies at $55 \mathrm{kHz}$ repetition (determined at 60 to $90 \mathrm{~W}$ ) and, by trend, at cutting velocities up to $300 \mathrm{~mm} / \mathrm{s}$. However, with increasing cutting velocity about $300 \mathrm{~mm} / \mathrm{s}$ we observe a decreasing ablation efficiency.

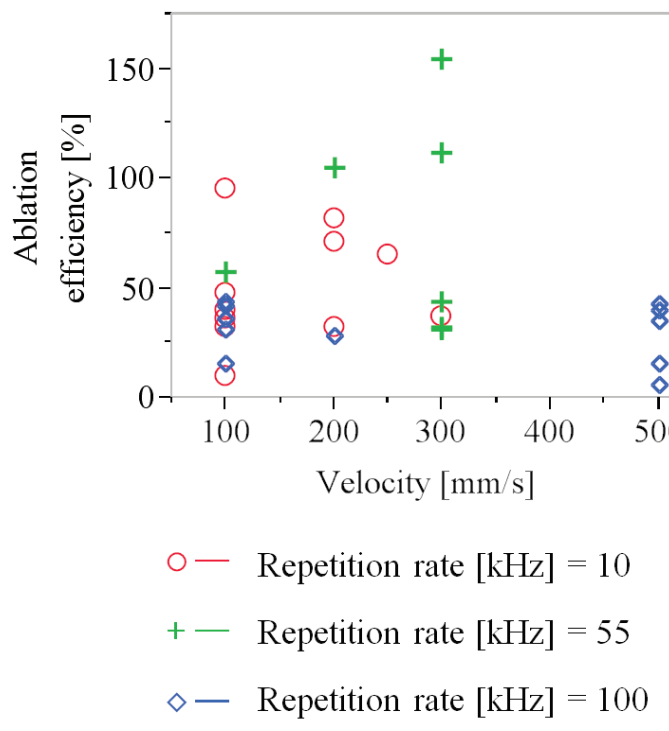

Fig 7 Ablation efficiency as a function of velocity grouped by repetition rate. Maximum ablation efficiency at $55 \mathrm{kHz}$ and $300 \mathrm{~mm} / \mathrm{s}$ for a given laser power regime.

Concluding the first part of the RSD, main influencing parameters are laser output power, cutting velocity and repetition rate. Highest productivity can be achieved at $80 \mathrm{~W}$ laser output power, $55 \mathrm{kHz}$ repetition rate and $300 \mathrm{~mm} / \mathrm{s}$ cutting speed.

\subsubsection{Quality measurements}

To analyse the quality of the cutting process, we have determined the breaking strength of the wafers and the chipping at the backside of the wafers.

The results of the corresponding RSD are shown in the contour plots 8 and 10, respectively, which have been derived from the $3 \mathrm{D}$-plots as described above.

Figure 8 shows the contours of the breaking strength versus laser power and velocity at a water pressure of 330bar and a repetition rate of $55 \mathrm{kHz}$. In general the function of the water jet is similar to a conventional gas jet. The higher the pressure the better is the removal of the molten material. In this case a high water pressure of 330 bar leads to high 
breaking strength. For lower water pressures the breaking strength turned out to be insufficient, being lower than $140 \mathrm{MPa}$. Please note that in this parameter regime the water pressure does not alter the chipping.

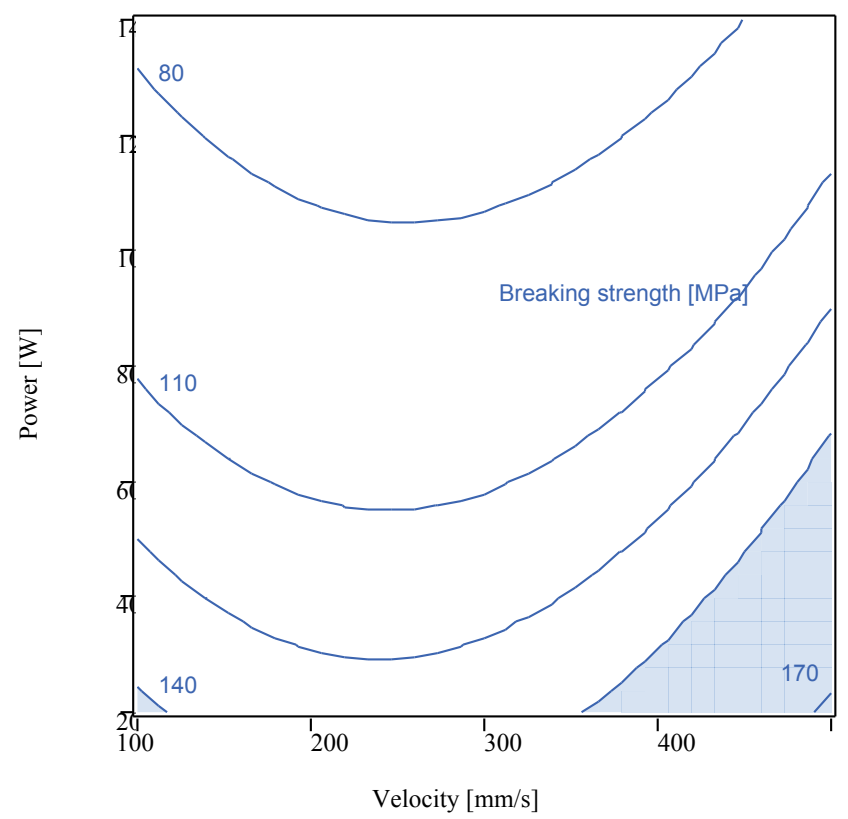

Fig. 8 Preferable breaking strength of about $140 \mathrm{MPa}$ as a function of velocity and power and a pre-set water pressure of 330 bar and repetition rate of $55 \mathrm{kHz}$.

In Figure 8, the blue shaded area highlights the preferable bending stress of at least $140 \mathrm{MPa}$, identifying a parameter range of cutting velocities above $350 \mathrm{~mm} / \mathrm{s}$ and laser powers in the range between 25 and $80 \mathrm{~W}$. Varying the repetition rate reveals no change in cutting results.

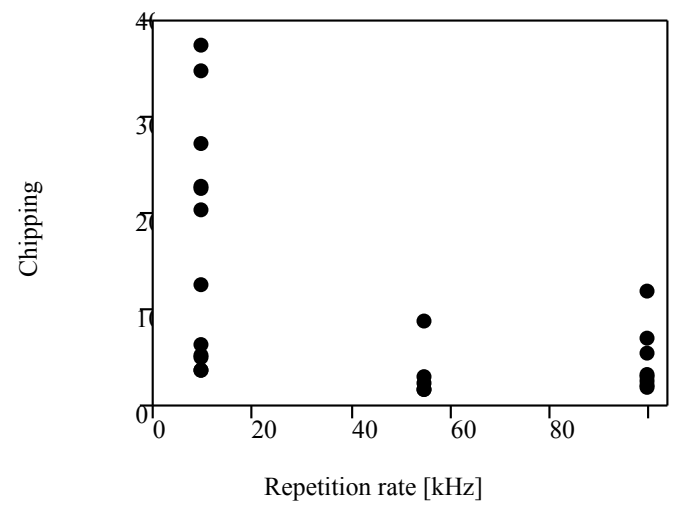

Fig. 9 Chipping as a function of repetition rate for various laser output powers, velocities and water pressures.

Since beside the breaking strength the chipping is of crucial importance, we have investigated the influence of the repetition rate on chipping. Figure 9 illustrates the chipping as a function of the repetition rate for various laser output powers and water pressures. We found that low repetition rates produce high chipping and, in addition, high heat affected zones, as described above. Repetition rates about $55 \mathrm{kHz}$ lead to less chipping due to lower pulse energies and pulse lengths of the used laser system.
Taking into account the productivity point of view, chipping values below 1000 outbreaks are preferable. Therefore, we limit the repetition rates in ranges from 55 to $100 \mathrm{kHz}$.

Combining the results for optimized breaking strength and chipping, only a small parameter range is identified to fulfill both requirements. Figure 10 shows the contour diagram for the parameter regime of laser output power and cutting velocity to obtain chipping values below 1000 (red highlighted area) and breaking strengths about $140 \mathrm{MPa}$ (blue marked area). The water pressure and the repetition rate were pre-set to $330 \mathrm{bar}$ and $55 \mathrm{kHz}$ based on desirable cutting results for the breaking strength and the chipping. Due to our measurement results we identified a small process window combining optimal breaking strength in conjunction with preferable chipping values, at laser powers in the range between 40 and $60 \mathrm{~W}$ at velocity regimes between 300 and $400 \mathrm{~mm} / \mathrm{s}$.

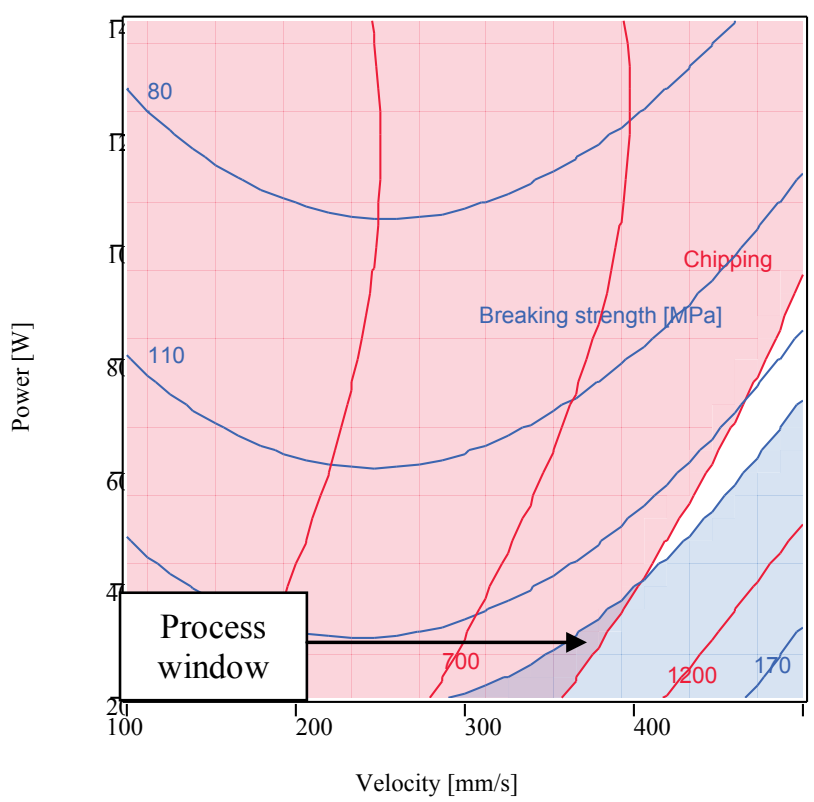

Fig. 10 Combining breaking strength and chipping (process window) as a function of velocity and laser output power for a pre-set water pressure of $330 \mathrm{bar}$ and repetition rate of $55 \mathrm{kHz}$.

Therefore, to optimize the parameter set, we identify a regime including cutting velocities of 300 to $400 \mathrm{~mm} / \mathrm{s}$, a laser power range from 40 to $60 \mathrm{~W}$ and a repetition rate of $55 \mathrm{kHz}$ in conjunction with a high water pressure at $330 \mathrm{bar}$. Table 4 lists the parameter sets to optimize quality and

Table 4 Optimized parameter sets

\begin{tabular}{lll}
\hline Parameter & Productivity & Quality \\
\hline Power [W] & 80 & 55 \\
Repetition rate [kHz] & 55 & 55 \\
Velocity [mm/s] & 300 & 400 \\
Water pressure [bar] & 215 & 330 \\
\hline
\end{tabular}

productivity.

Two further experiments for both optimized parameter sets demonstrate preferable results for effective cutting speed 
and chipping. As the quality of the thin silicon wafers, particularly the breaking strength, is the main evaluation criteria, the quality parameter set is the preferable parameter set, which in turn also satisfies the productivity requirements (Table 5).

Table 5 Results of the optimized parameter sets

\begin{tabular}{lll}
\hline Parameter & Productivity & Quality \\
\hline Breaking strength [MPa] & 100,9 & 148,1 \\
Chipping & 221,9 & 539,2 \\
Theoretical effective & 129,6 & 102,1 \\
speed [mm/s] & & \\
Ablation efficiency [\%] & 127 & 118 \\
\hline
\end{tabular}

Figure 11 illustrates the cutting result using the quality parameter set.

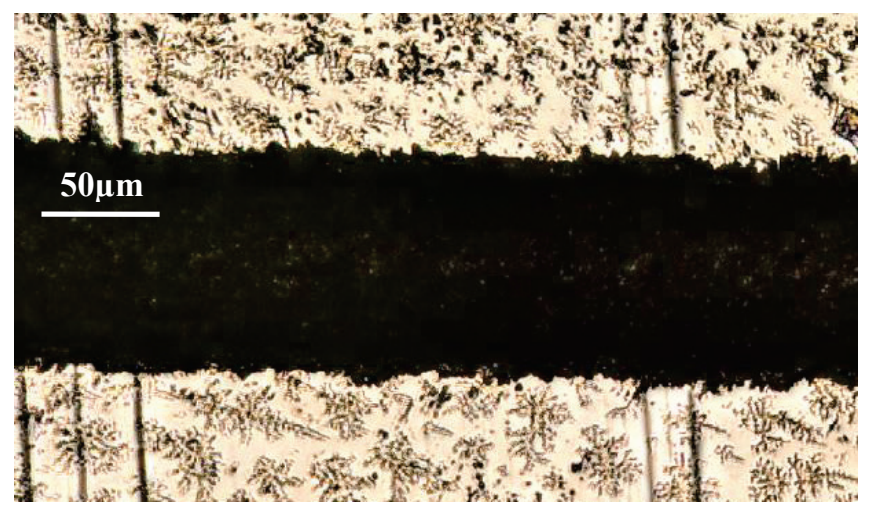

Fig. 11 Quality parameter set. The width of the cutting kerf of about $125 \mu \mathrm{m}$.

\section{Summary}

In summary, we have presented a comprehensive optimization study of water jet guided laser cutting of thin silicon wafers using $515 \mathrm{~nm}$ disc laser. The optimization study is based on a DOE approach, requiring only 33 experiments to identify an optimal parameter set to achieve both high quality and high productivity for the cutting process. Overall, the water pressure is the main influencing parameter for the quality. In turn, laser output power, cutting velocity as well as repetition rate are crucial parameters for the productivity.

\section{References}

[1] A. Pauchard: Precise thin metal cutting using the Laser MicroJet. In: Laser in electronics manufacturing, Proc. of the LEF Conf. Fürth, Germany (2009).

[2] A. Pauchard, M. Di Marco, B. Carron, G. Suruceanu, B. Richerzhagen, A. Brulé, and N. Kling: Recent developments in the cutting of ultra hard materials using water jet-guided laser technology. In: Proc. of the ALAC (2008).

[3] M. Di Marco, A. Pauchard, A. Spiegel, B. Richerzhagen, and N. Kling: Dicing of HB-LED Devices Embedded In Copper Substrates. In: Proc. of the ICALEO, Tennecula CA (2008).

[4] J. Hewett: Laser water jet cools and cuts in the material world. In: Optics and Lasers, Europe Issue 148 (2007).
[5] M. Gobet, S. Obi, M. Pavius, M. Takano, N. Vago, K. Lee, Y. Kozuki, and A. Pauchard: Implementation of short pulse lasers for wafer scribing and grooving applications. In: JLMN Vol. 5 (1), 16-20 (2010).

[6] A. Pauchard, N. Vago, and B. Richerzhagen: Experimental micromachining results using a UV laser with the Laser MicroJet. In: Proc. of the $3^{\text {rd }}$ Pacific Int. Conf. on Appl. of Lasers and Optics (2008).

[7] O. Sibally, F. Wagner, and B. Richerzhagen: Laser micromachining in microelectronics industry by water jet guided laser. In: LASE Vol. 5339 (2004).

[8] D. Perrottet, T.A. Mai, and B. Richerzhagen: Wet vs. Dry. In: Industrial Laser Solutions 5, 23-24 (2006).

[9] N.M. Dushkina and B. Richerzhagen: Dicing of Gallium Arsenide (GaAs) wafers with the Laser MicroJet: Challenges, Improvements and Safety Issues. In: Proc. of the ICALEO (2002).

[10]F. Wagner, A. Spiegel, and B. Richerzhagen: Water jet guided laser cutting using frequency doubled YAG lasers. In: Laser in electronics manufacturing, Proc. of the LEF Conf. Erlangen, Germany (2004).

[11]D. Perrottet, S. Amorosi, R. Housh, and B. Richerzhagen: Water jet guided lasers. In: Ceramic Industry Vol. 12 (2004).

[12]A. Seidl, B. Birkmann, B. Mackintosh, T. Grahl, J. Horzel, P. Roth, W. Schmidt, and I. Schwirtlich: Larger tube and wafer sizes: EFG on the cusp of a new generation. In: Proc. of the $21^{\text {st }}$ European Photovoltaic Solar Energy Conf. 988-991, Milan (2006).

[13]B. Birkmann, S. Guenther, F. Mosel, M. Müller, I. Westram, and A. Seidl: Growth of 200 micron thin EFG dodecagonal tubes: benefit of numerical simulation for process optimization. In: Proc. of the $23^{\text {rd }} \mathrm{Eu}-$ ropean Photovoltaic Solar Energy Conf., Valencia (2008).

[14]B. Richerzhagen, G. Delacrétaz, and R.P. Salathé: Complete Model to simulate the thermal defocussing of a laser beam focused in water. In: Optical Engineering, Vol. 35 (7), 2058-2066 (1996).

[15]A. Richter: Guided by water. In: Cutting Tool Engineering 8, 168 (2004).

[16]R. Hull: Properties of crystalline silicon. The Institution of Electrical Engineers. London (1999).

[17] S. Hopmann et al.: Comparison of Laser Chemical Processing and LaserMicroJet for structuring and cutting silicon substrates. In: Appl Phys A 95, 857-866 (2009).

[18]D.C. Montgomery: Design and Analysis of Experiments. John Wiley and Sons. New York (2001).

(Received: April 07, 2011, Accepted: May 21, 2011) 